\title{
Evidence for Capillary Waves on Dewetted Polymer Film Surfaces: A Combined X-ray and Atomic Force Microscopy Study
}

\author{
M. Tolan, O. H. Seeck, J.-P. Schlomka, and W. Press \\ Institut für Experimentelle und Angewandte Physik der Universität Kiel, Leibnizstraße 19, 24098 Kiel, Germany
}

\author{
J. Wang and S. K. Sinha \\ XFD/APS Argonne National Laboratory, 9700 South Cass Avenue, Argonne, Illinois 60439
}

\author{
Z. Li, M. H. Rafailovich, and J. Sokolov \\ Department of Materials Science and Engineering, State University of New York at Stony Brook, New York 11794-2275
}

(Received 12 May 1998)

\begin{abstract}
Surfaces of thin dewetted polyethylene-propylene films were investigated by atomic force microscopy (AFM) and $x$-ray scattering. The AFM images show the mesoscopic island structure but do not give information about the microscopic roughness of the polymer surface. Together with the AFM data we were able to identify capillary waves on the island surfaces by their specific power laws in the diffuse $\mathrm{x}$-ray scattering signal. The wave number spectrum of these waves is modified by a cutoff introduced by the van der Waals substrate-film interactions. [S0031-9007(98)07245-7]
\end{abstract}

PACS numbers: $68.15 .+\mathrm{e}, 61.41 .+\mathrm{e}, 68.45 . \mathrm{Gd}$

The spreading of simple liquids on solid substrates is well understood [1]. The equilibrium thickness depends on the van der Waals interactions between the solid and the liquid and is typically on the order of molecular dimensions. If the interfacial energies of the liquid at the solid and gaseous interfaces are larger than that of the dry substrate, dewetting occurs and droplets or islands begin to form. A large body work has been done concerning the mesoscopic structures that form during dewetting. Mostly polymer films were the focus of the investigations by optical microscopy or atomic force microscopy (AFM) (see, e.g., Refs. [2-5]). However, little is known about the microscopic structure of the surfaces on top of the islands of dewetted films. Previous investigations by Tidswell et al. [6] of capillary waves on liquid thin films that completely wet a solid surface show that long-range correlations on liquid surfaces can be identified by their specific x-ray scattering signals. Here we present the first quantitative study of capillary waves propagating on dewetted liquid polymer films. These films were in the initial capillary instability stages of dewetting where the sample surface is just roughened on a mesoscopic scale (islands), but a complete dewetting down to the substrate has not yet occurred.

From the theory of capillary waves it is known that the displacement-displacement correlation function $C(\mathbf{R})=$ $\langle z(\mathbf{r}) z(\mathbf{r}+\mathbf{R})\rangle_{\mathbf{r}}$ of a fluctuating interface, with $z(\mathbf{r})$ being the displacement at the lateral position $\mathbf{r}$, is given by [7-10]

$$
C_{\text {cap }}(R)=\frac{B}{2} K_{0}\left(q_{l, c} R\right),
$$

with $K_{0}(x)$ being the modified Bessel function of the first kind and $B=k_{B} T / \pi \gamma\left(B \sim 5-10 \AA^{2}\right.$ for most liquids and polymers at room temperature; $\gamma$ is the surface tension at the temperature $T$ ). The quantity $q_{l, c}$ is a "low wave vector cutoff" of the surface waves. This cutoff prevents the divergence of the thermally induced long-range fluctuations of the liquid surface and hence is strongly influenced by a background potential. The latter is simply given by gravity in the case of bulk liquids. For thin films the role of gravity is taken by the much stronger van der Waals interactions between the adsorbed film and the substrate. Hence long-range correlations are more effectively suppressed and the lower cutoff is given by $q_{l, c}=a / d^{2}$ with the film thickness $d$ and a characteristic length $a=\sqrt{A_{\text {eff }} / 2 \pi \gamma}$ which is on the order of $a \sim$ $5 \AA$ for most liquids ( $A_{\text {eff }}$ is the effective Hamaker constant) $[6,11,12]$.

In the case of a liquid that is in the initial stage of dewetting a substrate, mesoscopic structures ("islands") form. The question is whether capillary waves are still present on these islands and how they are influenced by the background potentials and the island structures. We have chosen the polymer polyethylene-propylene (PEP) as film material with a molecular weight $M_{W}=290 \times 10^{3}$ corresponding to a radius of gyration $R_{G} \approx 150 \AA$. Five films of thicknesses $d=48,88,107,201$, and $469 \AA$ were spun cast onto silicon wafers which were previously etched with hydrofluoric acid to thin the native oxide layer [13]. The samples were annealed for approximately one day at $180{ }^{\circ} \mathrm{C}$ under high vacuum conditions $(p \sim$ $10^{-6}$ mbar) to reach equilibrium and to evaporate the solvent. Afterwards they were cooled down to room temperature where all measurements took place. Since the glass transition temperature of PEP is $T_{G} \approx-62{ }^{\circ} \mathrm{C}$, the films are still expected to be in the liquid state [14].

The investigations were done in two steps: (i) AFM images of the PEP surfaces were taken with a Nanoscope III (Digital Instruments). These images reveal the characteristic island structures of dewetted films [3]. Since a liquid 
surface is fluctuating, it is clear that the specific properties of liquids cannot be measured by AFM. Therefore, (ii) $\mathrm{x}$-ray scattering experiments were performed at the synchrotron HASYLAB at DESY in Hamburg (Germany) at the bending magnet beam line E2. As it will be shown below, the x-ray scattering signal is sensitive to the microscopic part of the roughness, too, i.e., here to the capillary wave fluctuations. A monochromatic beam of wavelength $\lambda=1.00 \AA$ was used for the measurements. The scattering geometry with the in-plane components $q_{x}$ and $q_{z}$ of the scattering vector $\mathbf{q}$ is schematically depicted in the inset of Fig. 1 and was previously described in other works $[15,16]$. The vertical size of the slits gave a resolution of $\delta_{q x}=5 \times 10^{-5} \AA^{-1}$ at $q_{z}=0.2 \AA^{-1}$. Horizontally (in $y$ direction) the slits were wide open, leading to an effective integration over $q_{y}$. For each sample the reflectivity and transverse scans along $q_{x}$ for $q_{z}=0.15,0.20$, and $0.30 \AA^{-1}$ were taken.

Figure 2(A) shows the AFM data for the $d=107 \AA$ thick PEP film [17]. The island structure is visible. Quite similar structures were found on all surfaces. The island size is found to increase with increasing film thickness as observed previously by AFM [3] and according to theoretical predictions [18]. The line in Fig. 2(C) depicts the power spectral density (PSD) $L(q)$ as calculated from the AFM image. The PSD is the Fourier transform of the displacement-displacement correlation func-

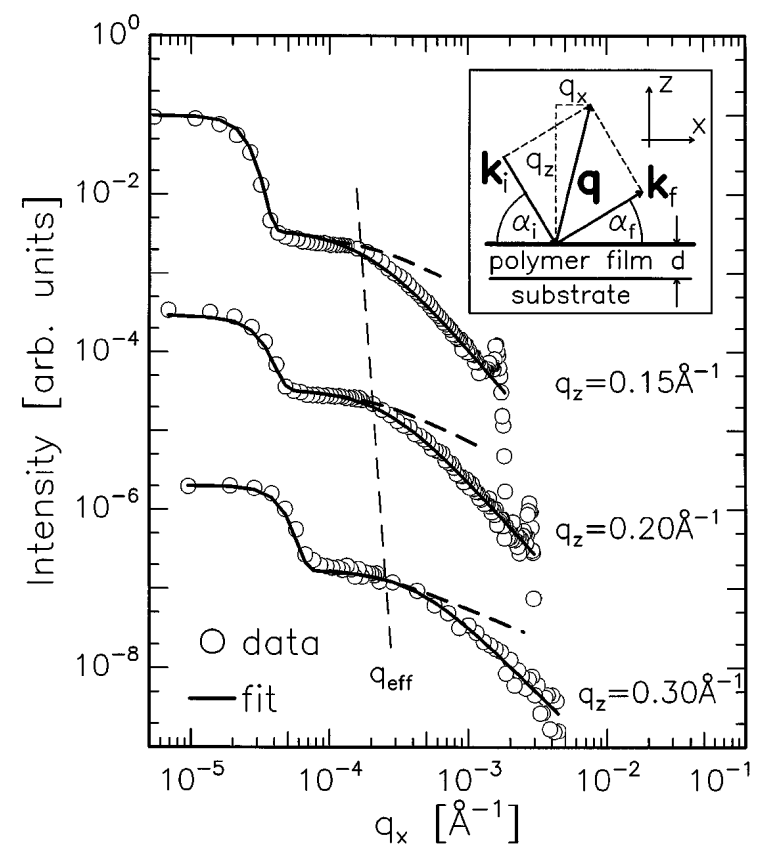

FIG. 1. Transverse scans along $q_{x}$ at $q_{z}$ values $0.15,0.20$, and $0.30 \AA^{-1}$ for a PEP film of thickness $d=201 \AA$ on silicon. The open circles are the data, and the lines are fits with the island cutoff model. The inclined vertical dashed line shows that the power-law region is separated from the flat part by a $q_{z}$-dependent cutoff $q_{\text {eff }}\left(q_{z}\right)$. The other dashed lines show calculations for capillary wave surfaces in the absence of islands. The inset depicts the scattering geometry. tion $C(R)$ and yields the distribution of wave numbers in the Fourier spectrum of the rough surface. Since no orientation is preferred, the respective angular average is plotted. A maximum at $q_{0}=3.1 \mu \mathrm{m}^{-1}$ can be seen which is caused by the regular mesoscopic structure of the islands (see below). The open circles in Fig. 2(C) show a numerically calculated PSD for the simulated island distribution as shown in Fig. 2(B) where circular islands, all of the same height and without microscopic roughness, were assumed. The result of the simulation and the image agree well, both in real and in reciprocal space. Figure 2(D) (line and open circles) shows the corresponding PSD's $L\left(q_{x}\right)$, but now averaged over $q_{y}$ instead of the angular dimension. This quantity corresponds essentially to what is measured in an x-ray experiment (crosses). It is worth noting that this average removes the maximum at $q_{0}$.

The discussion above shows that the AFM data can be fully explained by the mesoscopic island structure but further details are invisible. The main reason for this is that details on an atomic scale of fluctuating interfaces are hard to observe with an AFM where a tip is moved over the surface. Here scattering techniques have significant advantages.

To calculate the off-specular $\mathrm{x}$-ray scattering from a dewetted PEP surface, one needs the real-space
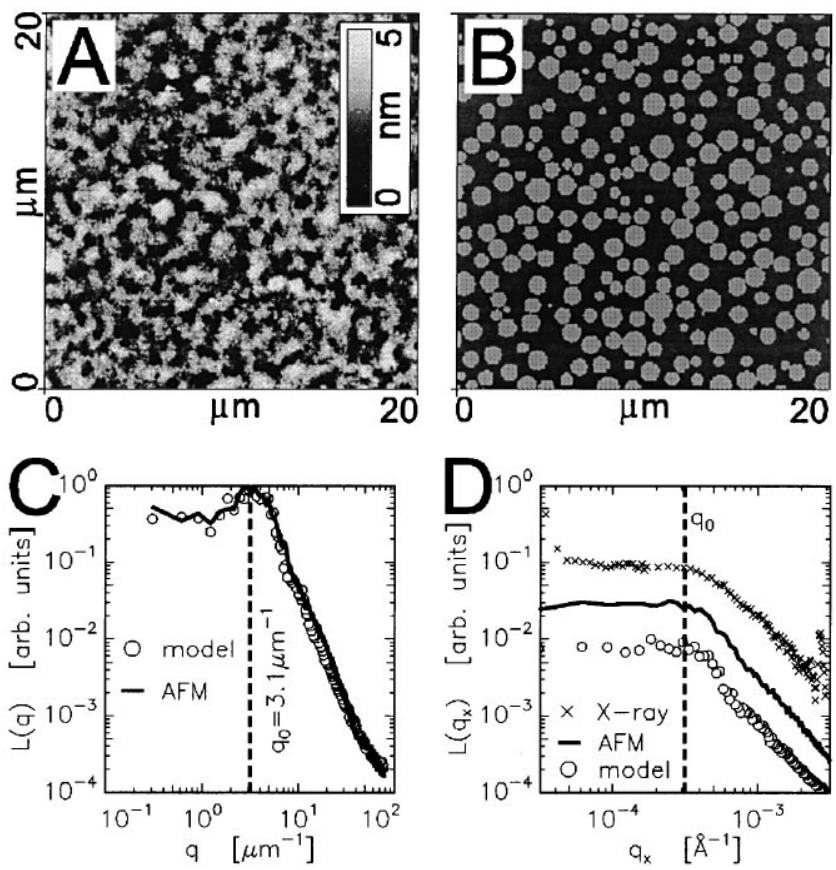

FIG. 2. (A) AFM image of a dewetted PEP surface for a film of thickness $d=107 \AA$. (B) Sketch of a surface possessing a random arrangement of circular islands. (C) Line: PSD $L(q)$ as obtained from a 2D Fourier transform of the AFM image and a subsequent angular average. Open circles: PSD obtained by Fourier transforming the arrangement shown in image (B). (D) Line: PSD $L\left(q_{x}\right)$ averaged over $q_{y}$ as calculated from the AFM image. Open circles: PSD $L\left(q_{x}\right)$ averaged over $q_{y}$ as calculated with the arrangement shown in (B). Crosses: $x$-ray data obtained with a coarse resolution in $q_{y}$ direction. 
correlation function $C(R)$ of capillary waves on islands. We have chosen the ansatz $C(R)=C_{\text {cap }}(R)+C_{\text {is }}(R)$ which is reasonable because one may separate the displacement term $z(\mathbf{r})$ into a capillary wave and an island part; i.e., $z(\mathbf{r})=z_{\text {cap }}(\mathbf{r})+z_{\text {is }}(\mathbf{r})$, where the two fluctuations are independent. For the island correlation function the following simple form is able to explain all major features of the scattering:

$$
C_{\text {is }}(R)=\sigma_{\text {is }}^{2} \exp (-\Delta q R) \cos \left(q_{D} R\right),
$$

where $q_{D}=2 \pi / D$ is the periodicity given by the average size $D$ of the islands, $\Delta q$ defines the width of the size distribution in reciprocal space, and $\sigma_{\text {is }}$ is the "rms roughness" caused by the islands. It is obvious that both $\Delta q$ and $\sigma_{\text {is }}$ are island size and thus film thickness dependent also (for details see Ref. [3]). The Fourier transform of Eqs. (1) and (2) yields the PSD $L(q)$ of an island covered surface possessing capillary waves. An analytic discussion of the Fourier transform of $C_{\text {is }}(R)$ reveals that the PSD exhibits a peak for a narrow size distribution, or more quantitatively for $q_{D}>\Delta q$. In the reverse case, i.e., if the mean value of the reciprocal island size $q_{D}$ is smaller than the width $\Delta q$ of the respective distribution, the surface appears essentially random in all directions and the PSD does not show a peak. The island part is again very similar to the functions shown in Fig. 2(C) where the location $q_{0}$ of the maximum is given by $q_{0}=\sqrt{q_{D}^{2}-(\Delta q)^{2}}$. An important feature of the curves shown in Figs. 2(C) and 2(D) is the slope of the respective PSD's for $q>q_{0}$ and $q_{x}>q_{0}$, respectively. This slope is $\approx-3$ for the numerically obtained PSD and that calculated from the AFM image and $\approx-2$ for the $q_{y}$ averaged functions $L\left(q_{x}\right)$ depicted in Fig. 2(D). As it can be proven analytically, these features are also correctly described by Eq. (2). Hence $C_{\text {is }}(R)$ is a statistically complete real space description of the AFM images.

Since the scattering is proportional to the Fourier transform of $\exp \left\{q_{z}^{2} C(R)\right\}[19,20]$ and here $C_{\text {cap }}(R)+$ $C_{\text {is }}(R)$, we obtain the following scattering function $S(\mathbf{q})$ of the PEP surface in Born approximation

$$
\begin{aligned}
S\left(q_{x}, q_{z}\right)= & A \frac{(\Delta \varrho)^{2}}{q_{z}^{2}} \exp \left\{-q_{z}^{2}\left(\sigma_{\text {cap }}^{2}+\sigma_{\text {is }}^{2}\right)\right\} \\
& \times S_{\text {cap }}\left(q_{x}, q_{z}\right) * S_{\text {is }}\left(q_{x}, q_{z}\right),
\end{aligned}
$$

where $\sigma_{\text {cap }}$ is the roughness due to capillary waves as defined in Ref. [19], $\Delta \varrho$ is the PEP/air scattering length density contrast, $S_{\text {cap }}\left(q_{x}, q_{z}\right)=$ $\int \exp \left\{q_{z}^{2} C_{\text {cap }}(X)+i q_{x} X\right\} d X \quad$ and $\quad S_{\text {is }}\left(q_{x}, q_{z}\right)=$ $\int \exp \left\{q_{z}^{2} C_{\text {is }}(X)+i q_{x} X\right\} d X$ are separately $\left(q_{x}, q_{z}\right)$ dependent scattering functions, from capillary waves and islands alone, respectively, and (*) denotes a convolution in $q_{x}$. Since the measurements have shown that all diffuse scattering stems essentially from the dewetted PEP film surfaces, the scattering from the very smooth silicon substrates was neglected in Eq. (3).
A discussion of Eq. (3) yields power-law expressions for $S\left(q_{x}, q_{z}\right)$ which are valid for $q_{x}>q_{\text {eff }}\left(q_{z}\right)=\left(q_{l, c}^{2}+\right.$ $\left.\eta^{2} q_{0}^{2}\right)^{1 / 2}$ with $\eta=B q_{z}^{2} / 2$. Furthermore, the particular $q_{z}$-dependent capillary wave exponent [19] changes for thin dewetted films from $S \propto q_{x}^{\eta-1}$ to $S \propto q_{x}^{\eta-2}$.

Figures 1 and 3 depict the data (circles) and fits (lines) of transverse scans taken along the direction $q_{x}$ (see inset of Fig. 1). The data shown in Fig. 3 were measured at $q_{z}=0.2 \AA^{-1}$. The region $q_{x}<5 \times 10^{-5} \AA^{-1}$ contains the specular peak which was modeled as a Gaussian. The diffuse scattering, i.e., the intensity measured for $q_{x}>5 \times 10^{-5} \AA^{-1}$, was fitted with the expression given by Eq. (3). Two regions can be identified: (i) A rather flat region close to the specular peak. The size of this region decreases with increasing film thickness. (ii) A powerlaw region where the diffusely scattered intensity is proportional to $q_{x}^{\eta-2}$. Figure 1 shows that the exponent is a function of $q_{z}$ and the fits (lines) reveal the $q_{z}$-dependent exponents typical of capillary waves. However, due to the islands the additional correlation function $C_{\mathrm{is}}(R)$ yields a $q_{x}^{\eta-2}$ behavior instead of the usual pure capillary wave form $q_{x}^{\eta-1}$ [19] (see dashed lines in Fig. 1). The inclined vertical dashed line emphasizes that the cutoff $q_{\text {eff }}$ is a function of $q_{z}$, too, as anticipated by the calculations of

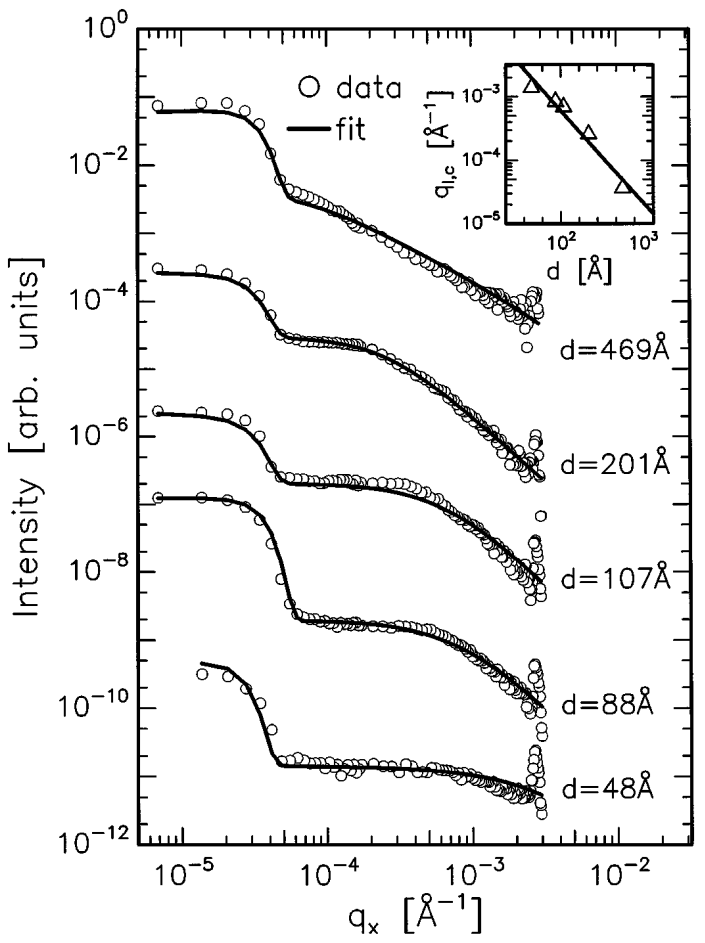

FIG. 3. Diffuse scattering from PEP films on silicon substrates with film thicknesses $d=48, \ldots, 469 \AA$. Transverse scans (open circles) for fixed $q_{z}=0.2 \AA^{-1}$ are shown. The lines are fits to the data. The inset depicts a log-log plot of the lower capillary wave cutoff $q_{l, c}$ vs $d$ (open triangles $q_{l, c}$ obtained from the diffuse scattering fits; line: linear fit). The Yoneda peak at $q_{x} \approx 3 \times 10^{-3} \AA^{-1}$ was excluded from the data analysis. 
the scattering above. Only for the thickest film the whole diffuse scattering is essentially determined by the powerlaw region which shows a $q_{x}^{\eta-1}$ behavior. Thus, for this thickness already free capillary waves which propagate on the liquid PEP surface are found.

We emphasize here again that the fits shown in Fig. 1 confirm the $q_{z}$ dependence of the exponent in the powerlaw region. This particular form is a fingerprint of a logarithmically diverging correlation function; i.e., it proves unambiguously the presence of capillary waves on the liquid PEP surfaces $[19,20]$.

The inset of Fig. 3 depicts a log-log plot of $q_{l, c}$ vs the film thickness. The straight line with slope $-1.6 \approx$ -2 suggests that a van der Waals cutoff $q_{l, c}=a / d^{2}$ with $a \approx 7 \AA$ can be identified by our data. From the nominal parameters for PEP, $A_{\text {eff }} \sim 10^{-18}-10^{-19} \mathrm{~J}$ for the effective Hamaker constant and $\gamma=30 \mathrm{dyn} / \mathrm{cm}^{2}$ for the surface tension (for a detailed discussion see Ref. [3] and references therein), a value of $a=7-20 \AA$ may be calculated, again showing a good agreement between data and theory.

The theory of Brochard et al. [18] for resonant capillary modes which are present at the prerupture stage of an unstable film in the initial stage of dewetting yields a power law behavior for the respective resonant wave number. If we assume that our dewetted structures have resulted from the evolution of these amplified fluctuations, the wave number $q_{0}$ of the maximum of the PSD [see Fig. 2(C)] may be estimated by $q_{0} \propto d^{-m}$. However, we are aware of the fact that this is only a crude approximation. The analysis of our data suggests $m \approx 1$. Since the error bars are quite large, the theoretical value of $m=3 / 2$ is still within the accuracy of our experiment.

In summary, we have presented the first study of thermally induced height-height fluctuations on polymer surfaces which are on the initial stage of dewetting. Our results suggest the existence of capillary waves on the island substructures. The propagation of these surface waves is suppressed by the background van der Waals potential and superimposed over the island structure. The two effects were separated in a most direct and unambiguous manner by simultaneous AFM and x-ray scattering measurements. Further work may help to understand the microscopic mechanism of dewetting since the wave number spectrum of capillary waves is very sensitive to the microstructure of the films and the specific form of the substrate/adsorbate interactions.

M. T. thanks the German Science Foundation (DFG Contract No. To 169/2-1) and the Advanced Phonton
Source for financial support. This work was partially supported by DOE under BES, Contract No. W-31-109ENG-38.

[1] P. G. de Gennes, Rev. Mod. Phys. 57, 827 (1985).

[2] G. Reiter, Phys. Rev. Lett. 68, 75 (1992).

[3] W. Zhao, M.H. Rafailovich, J. Sokolov, L. J. Fetters, R. Plano, M. K. Sanyal, S. K. Sinha, and B. B. Sauer, Phys. Rev. Lett. 70, 1453 (1993).

[4] C. Redon, F. Brochard-Wyart, and F. Rondelez, Phys. Rev. Lett. 66, 715 (1991).

[5] P. Müller-Buschbaum, P. Vanhoorne, V. Scheumann, and M. Stamm, Europhys. Lett. 40, 655 (1997).

[6] I. M. Tidswell, T. A. Rabedeau, P. S. Pershan, and S.D. Kosowsky, Phys. Rev. Lett. 66, 2108 (1991).

[7] F.P. Buff, R. A. Lovett, and F.H. Stillinger, Phys. Rev. Lett. 15, 621 (1965).

[8] S. Dietrich, J. Phys. Condens. Matter 8, 9127 (1996).

[9] J. Jäckle and K. Kawasaki, J. Phys. Condens. Matter 7, 4351 (1995).

[10] A. Braslau, P.S. Pershan, G. Swislow, B. M. Ocko, and J. Als-Nielsen, Phys. Rev. A 38, 2457 (1988).

[11] J. Israelachvili, Intramolecular Surface Forces (Academic Press Inc., New York, 1992), 2nd ed.

[12] Sferrazza, C. Xiao, R. A. L. Jones, D. G. Bucknall, J. Webster, and J. Penfold, Phys. Rev. Lett. 78, 3693 (1997).

[13] The film thicknesses were determined by the analysis of specular x-ray reflectivity data. These data also reveal that the substrate/polymer interface is very smooth.

[14] The glass transition temperature $T_{G}$ may, however, be strongly film thickness dependent [see, e.g., W.E. Wallace, J.H. van Zanten, and W.L. Wu, Phys. Rev. E 52, R3329 (1995)].

[15] P. Müller-Buschbaum, S. A. O'Neill, S. Affrossman, and M. Stamm, Macromolecules 31, 3686 (1998).

[16] P. Müller-Buschbaum, M. Casagrande, J. Gutmann, T. Kuhlmann, M. Stamm, G. von Krosigk, U. Lode, S. Cunis, and R. Gehrke, Europhys. Lett. 42, 517 (1998).

[17] Here we note that the height of the islands does not reflect the actual thickness of the film because the sample surface is "roughened" on a mesoscopic scale (island structure) while no complete dewetting of the substrate has occurred. Figure 2(B) is an oversimplified illustration of this scenario which could serve as a model for the lateral structure of the sample.

[18] F. Brochard-Wyart, C. Redon, and C. Sykes, C.R. Acad. Sci. Ser. Gen., Ser. 2 19, 314 (1992).

[19] M. K. Sanyal, S. K. Sinha, K. G. Huang, and B. M. Ocko, Phys. Rev. Lett. 66, 628 (1991).

[20] S. K. Sinha, E. B. Sirota, S. Garoff, and H. B. Stanley, Phys. Rev. B 38, 2297 (1988). 